\title{
Long term evaluation of mental fatigue by Maastricht Questionnaire in patients with OSAS treated with CPAP
}

\author{
P. Carratù1, G. Karageorgiou1, P. Bonfitto2, G. Di Gioia1, \\ D. Lacedonia1, M.P. Foschino Barbaro2, O. Resta1
}

ABSTRACT: Long term evaluation of mental fatigue by Maastricht Questionnaire in patients with OSAS treated with CPAP. P. Carratù, G. Karageorgiou, P. Bonfitto, G. Di Gioia, D. Lacedonia, M.P. Foschino Barbaro, O. Resta.

Background. Patients with obstructive sleep apnoea syndrome (OSAS) suffer from disrupted sleep. Impaired nightly sleep leads to increase physical and mental fatigue. The effect of long term continuous positive airway pressure (CPAP) on mental fatigue in OSAS patients, assessed by Maastricht Questionnaire (MQ), has not been investigated yet.

Methods. In order to evaluate the role of CPAP in improving mental fatigue of patients with OSAS, we studied 35 patients ( 26 males, age $<65$ years at the time of the diagnosis) affected by OSAS, established by polysomnography (PSG). Patients were divided into two groups; 19 subjects (15 males), who refused CPAP therapy, and 16 patients (11 males) well matched for sex, age, body mass index (BMI), neck circumference, duration of follow up, and severity of disease, who had been treated with CPAP for at least two years.

Results. All patients had severe OSAS with Respiratory Disturbance Index (RDI), of $48 \pm 20.9$ (range 22-90) and
61.48 \pm 18.6 (range 34-101) respectively, for group one (untreated patients) and group two (CPAP treatment). In addition, all patients had severe impairment of mental fatigue and of daytime sleepiness, demonstrated by high values of MQ score $(32.17 \pm 15.33$ and $37.36 \pm 12.4$, respectively) and Epworth Sleepiness Scale (ESS) $(14.21 \pm 4.77$ and $15.06 \pm 6.07$ respectively). There was no statistical significant difference in the group one at baseline and after follow-up, in terms of BMI, MQ score, ESS, and RDI. In the CPAP group (group two), the patients reported a significant improvement of the quality of their mental health (MQ 37.36 \pm 12.4 vs. $16.41 \pm 9.02 ; p<0.0001)$ and sleepiness (ESS $15.06 \pm 6.07$ vs. $4.13 \pm 3.93 ; p<0.0001$ ) with a stable BMI. There was significant correlation between the severity of sleep apnoea, expressed as RDI, and MQ at admission compared to at the end of follow-up $(r=0.4, p<0.05)$.

Conclusions. This study demonstrates an evident deterioration of mental fatigue in patients with OSAS, directly correlated to the severity of nocturnal disorder breathing; however supports the hypothesis that long term CPAP therapy significantly improves sleepiness and mental fatigue.

Monaldi Arch Chest Dis 2007; 67: 1, 6-9.

Keywords: Mental fatigue, Maastricht Questionnaire, obstructive sleep apnea syndrome, continuous positive airway pressure.

1 Institute of Respiratory Disease, Department of Clinical Methodology and Medical Surgery Technology, University of Bari,

2 Institute of Respiratory Disease, University of Foggia, Italy.

Correspondence: Prof. Onofrio Resta, MD; Department of Clinical Methodology and Medical Surgery Technology, University of Bari, Italy, Piazza G. Cesare 11, 70124 Bari, Italy; e-mail: oresta@pneumol.uniba.it

\section{Introduction}

Patients with obstructive sleep apnoea syndrome (OSAS) suffer from disrupted sleep with frequent electroencephalogram (EEG) arousals and elevation of sympathetic activity [1]. Impaired nightly sleep leads to increase physical and mental fatigue. A recent study showed that patients with mild, moderate OSAS have highly exhausted mental status, evaluated by a modified version of the Maastricht Questionnaire (MQ) [2], compared to patients without OSAS. To our knowledge, no study has investigated the effects of long term continuous positive airway pressure (CPAP) on the mental fatigue in OSAS patients, assessed by the Maastricht questionnaire. Furthermore, the long term variation of mental fatigue in OSAS patients without treatment has not been estimated yet. In this controlled study, we investigated the effect of long term CPAP therapy on mental fatigue in a group of patients with OSAS compared to a well matched group (in terms of sex, age, body mass index [BMI], degree of disease and follow-up) of untreated patients affected by OSAS. 


\section{Material and Methods}

\section{Patients}

The study was conducted at the Sleep Laboratory of the Respiratory Diseases Institute, University of Bari, School of Medicine, from December 1992 to January 2005. We enrolled 35 patients with OSAS, age $<65$ years at the time of the initial diagnosis, assessed by polysomnography (PSG). We excluded patients with illness, such as depression, neurological degenerative diseases, severe heart diseases, chronic obstructive pulmonary disease, and renal failure, in order to avoid other causes of fatigue. The patients were also excluded if they had Periodic Limb Movement (PLM) disorders.

We divided the patients into two groups, matched for sex, age, BMI, neck circumference, length of follow up and severity of disease. The first group of 19 patients (group one) refused CPAP therapy. The second group (group two) of 16 patients was treated with CPAP for at least two years. The polysomnography was performed according to the protocol of our sleep laboratory [3]. The diagnosis of OSAS was made according to ASDA criteria [4]. CPAP indication was made according to the guidelines of Loube et al. [5]. We included into group one only patients who did not undergo surgical maxillo facial or ear nose and throat intervention. We enrolled into group two patients who correctly used CPAP for at least 2 years, at least 4 hours per night, for at least 6 nights per week. In this regard, we directly verified these parameters from ventilator prothesis. No patient in either of the two groups was ever treated with anti-depressive or sedative drugs in the past.

\section{Anthropometric data}

Weight was measured to the nearest kilogram and height to the nearest centimetre. Body mass index (BMI) was calculated as the weight $(\mathrm{kg})$ divided by the square of height (m). Neck circumference was determined at the level of the cricothyroid membrane.

\section{Measurement of mental fatigue and somnolence}

The individual mental fatigue and the somnolence were simultaneously measured by Form B of Maastricht Questionnaire (MQ) and the Epworth Sleepiness Scale (ESS), respectively, at time of initial diagnosis and during the follow up. We made an Italian computer version of the Form B of Japanese MQ [2] which consists of 21 items referring to excess fatigue, loss of vigour, increased irritability, and feelings of demoralisation. Each statement might be answered Never, Seldom, Sometimes or Always, using a four point scale of probability $(0,1,2,3)$. The scale score was obtained by the calculation of the numbers selected for the 21 questions (table 1). According to Hayakawa et al. [2] a higher MQ score was considered as stronger mental fatigue. The ESS was obtained by asking the subjects whether they had daytime sleepiness in 8 common situations using a four point scale of probability $(0,1,2,3)$ according to Johns et al. [7]. A score <10 was considered as normal.

\section{Statistical analysis}

Data is reported as mean $\pm \mathrm{SD}$. We considered significant a $p$-value of $\leq 0.05$. The continuous variables were analysed using a two tails Students'

Table 1. - Maastricht Questionnaire modified by Hayakava et al.

Maastricht Questionnaire. Answers might be NEVER, SELDOM, SOMETIMES, ALWAYS $(0,1,2,3)$

Do you often feel tired?

Do you often have trouble falling asleep?

Do you wake up repeatedly during the night?

Do you feel weak all over?

Do you have the feeling that you have not been accomplishing much lately?

Do you have the feeling that you cannot cope with everyday problems as well as you used to?

Do you believe that you have come to a 'dead end'

Do you lately feel more listless than before?

I enjoy sex as much as ever

Have you experienced a feeling of hopelessness recently?

Does it take more time to grasp a difficult problem than it did a year ago?

Do little things irritate you more than they used to?

Do you feel you want to give up trying?

I feel fine

Do you sometimes feel that your body is like a battery that is losing its power?

Would you want to be dead at times?

Do you have the feeling these days that you just do not have what it takes any more?

Do you feel dejected?

Do you feel like crying sometimes?

Do you ever wake up with a feeling of exhaustion and fatigue?

Do you have increasing difficulty in concentrating on a single subject for long? 
$t$-test. The categorical values were analysed using the $\chi$-square test. The correlations between variables were analysed using the Spearman rank correlation test.

\section{Results}

Thirty-five patients participating to this study showed clear signs of disease. Table 2 shows the characteristics of the two groups at the time of initial diagnosis. As shown, all patients had severe OSAS with RDI of 48,0 $\pm 20,9$ (range 22-90) and $61,48 \pm 18,6$ (range 34-101) respectively, for group one (untreated patients) and group two (CPAP treatment). In addition, all patients had severe impairment of mental fatigue and of daytime sleepiness, demonstrated by high values of MQ score $(32,17 \pm 15,33$ and $37,36 \pm 12,4$, respectively) and ESS (14,21 $\pm 4,77$ and $15.06 \pm 6.07$, respectively). There was no statistical difference between the two groups with regard to all the parameters observed, including mean of age, BMI, and period of assessment (table 2).

Table 3 shows the differences among BMI, RDI, MQ score, and ESS at admission and after the follow up period in the group of the untreated patients (group one). There was no statistical significant difference among patients of group one, before and after follow-up, in terms of BMI (38.62 \pm 8.59 vs. $38.96 \pm 8.96)$, MQ score $(32.17 \pm 15.33$ vs. $36.2 \pm 11.7), \operatorname{ESS}(14.21 \pm 4.77$ vs. $14.84 \pm 6.27)$, and RDI (48.02 \pm 20.98 vs. $53.38 \pm 19.04)$. In the CPAP group, as shown in table 4 , the patients reported a significant improvement of the quality of their mental health (MQ 37.36 \pm 12.4 vs.16.41 +9.02; $p<0.0001$ ) and sleepiness (ESS 15.06 \pm 6.07 vs. $4.13 \pm 3.93 ; p<0.0001)$ with a stable BMI. There was significant correlation between the severity of sleep apnoea, expressed as Respiratory Disturbance Index (RDI), and MQ at admission compared to at the end of follow-up $(r=0.4, p<0,05)$.

Out of the 16 patients of group two, 13 improved their MQ score. Among the 19 patients of the group one, MQ did not change in 10 individuals, while worsened in 5 patients, and improved in 4. ESS increased in almost all the patients treated with CPAP, while there was no variation in 8 subjects, impaired in 6 and improved in 4 patients of group one.

Finally, there was not significant correlation among basal RDI, improvement of MQ score and

Table 2. - Baseline characteristics of the two groups expressed as mean $\pm \mathrm{SD}$

\begin{tabular}{lccc}
\hline & Non CPAP & CPAP & $p$-value \\
\hline Number & 19 & 16 & $\mathrm{~ns}$ \\
M/F & $15 / 4$ & $11 / 5$ & $\mathrm{~ns}$ \\
Age & $53.53 \pm 8.17$ & $53.06 \pm 8.84$ & $\mathrm{~ns}$ \\
BMI & $38.62 \pm 8.59$ & $40.85 \pm 8.92$ & $\mathrm{~ns}$ \\
RDI & $48.02 \pm 20.98$ & $61.48 \pm 18.66$ & $\mathrm{~ns}$ \\
MQ & $32.17 \pm 15.33$ & $37.36 \pm 12.4$ & $\mathrm{~ns}$ \\
ESS & $14,21 \pm 4,77$ & $15.06 \pm 6.07$ & $\mathrm{~ns}$ \\
Follow up time (years) & $6.79 \pm 2.20$ & $6.22 \pm 2.21$ & \\
\hline
\end{tabular}

Table 3. - Anthropometric, MQ, ESS score, and RDI before and after follow up in patients not in CPAP treatment, expressed as mean $\pm \mathrm{SD}$

Non CPAP group

\begin{tabular}{lccc}
\hline 19 & Before follow up & After follow up & p-value \\
\hline BMI & $38.62 \pm 8.59$ & $38.96 \pm 8.96$ & $\mathrm{~ns}$ \\
MQ & $32.17 \pm 15.33$ & $36.2 \pm 11.7$ & $\mathrm{~ns}$ \\
ESS & $14.21 \pm 4.77$ & $14.84 \pm 6.27$ & $\mathrm{~ns}$ \\
RDI & $48.02 \pm 20.98$ & $53.38 \pm 19.04$ & $\mathrm{~ns}$ \\
\hline
\end{tabular}

Table 4. - Anthropometric, MQ, ESS score before and after follow up in patients with CPAP treatment, expressed as mean $\pm \mathrm{SD}$

\section{CPAP group}

\begin{tabular}{lccc}
\hline $\mathbf{1 6}$ & Before follow up & After follow up & $p$-value \\
\hline BMI & $40.85 \pm 8.92$ & $40.31 \pm 8.31$ & $\mathrm{~ns}$ \\
MQ & $37.36 \pm 12.4$ & $16.41 \pm 9.02$ & 0.0001 \\
ESS & $15.06 \pm 6.07$ & $4.13 \pm 3.93$ & 0.0001 \\
\hline
\end{tabular}


ESS in CPAP group, while there was a significant correlation between basal MQ score and the degree of improvement, after CPAP $(\mathrm{r}=0,9, p<0,0001)$.

\section{Discussion}

This study shows that patients affected by severe OSAS have an elevated mental fatigue which might be improved with the use of long term CPAP therapy. Among them, individuals with more severe OSAS reported a higher mental fatigue. In patients not treated with CPAP, mental fatigue did not change during the time of observation, or showed a tendency to deteriorate according to the severity of OSAS. We also observed similar results at evaluation of the daytime sleepiness.

Previous studies reported a large number of neuropsychiatric disturbances in subjects with OSAS $[8,9]$, while a recent paper of Hayakawa et al. [2], investigating a population of OSAS with different degrees of severity, showed that these patients demonstrated a higher mental fatigue, verified by Maastricht Questionnaire, with respect to a matched control group. However, these authors, found lower MQ scores in patients affected by severe OSAS, and hypothesised that patients with more severe OSAS were characterised by neuropsychiatric disturbances (such as attention loss), that could interfere with the ability to fully appreciate the quality of their mental status and could justify the lack of collaboration answering the questionnaire, and the relation between RDI and MQ score. This data is different from the Tousignant paper [10], which demonstrated a correlation between RDI and mental health state, resulting in higher severity of disease, elevated mental fatigue and lower health status. Tousignant's and our study, which consists in an Italian computer version of the Form B of Japanese MQ, were conducted retrospectively and, therefore, there might be a bias of recall about the pre-treatment mental status and health state. In addition, one important finding of our study was established by the high improvement of MQ score and mental fatigue, in patients treated with long term CPAP (mean follow up was $>6$ years), compared to the OSAS group not treated with CPAP, and matched for gender, age, BMI, severity of RDI; suggesting that the length of ventilator therapy plays a crucial role in decreasing MQ score. Furthermore, MQ score improvement, after CPAP therapy, was not related to the baseline RDI, but to the baseline MQ. A reliable limit of the study is represented by the adaptation of the Maastricht Questionnaire, initially applied and validated for cardiac Caucasian subjects, to a Japanese version, in order to study Japanese OSAS patients. We subsequently adapted this questionnaire from the Japanese form, directly to an Italian population of OSAS, although there is more of an ethnic difference in the two samples, than a patho-physiologic discrepancy. However previous studies showed an improvement of the neuropsychological parameters and quality of life with shorter follow up [11], to our knowledge, this is the first study in which the Maastricht Questionnaire test was assessed to evaluate the long term effects of CPAP on mental fatigue in OSAS patients. We did not find a significant worsening in MQ, ESS, and RDI in untreated subjects, probably due to the long-lasting history of disease, likely due to the high RDI score at admission. On the other hand, MQ did not play an active role in determining a worsening of the observed parameters, neither the increase of age seems to be important, since we did not observe any correlation between age and MQ. While, in patients treated with CPAP, MQ and ESS score significantly improved.

In conclusion, this study demonstrates a significant deterioration of mental fatigue in patients with OSAS, directly correlated to the severity of nocturnal disorder breathing, supporting the hypothesis that long term CPAP therapy may improve significantly the sleepiness and the mental fatigue.

\section{References}

1. Watanabe T, Mano T, Iwase S, et al. Enhanced muscle sympathetic nerve activity during sleep apnea in the elderly. J Auton Nerv Sys 1992; 37: 223-226.

2. Hayakawa T, Osamu Fujita, Kazuya Ishida, et al. Evaluating mental fatigue in patients with obstructive sleep apnea syndrome by the Maastricht Questionnaire. Psychiatry and Clinical Neurosiences 2002; 56: 313-314.

3. Resta O, Foschino-Barbaro MP, Legari G, et al. Sleeprelated breathing disorders, loud snoring and excessive daytime sleepiness in obese subjects. Internat J Obesity 2001; 25: 669-675.

4. Sleep Related Breathing Disorders in Adults: Recommendations for Syndrome Definition and Measurement Techniques in Clinical Research. Sleep 1999; 1; 22: 616-627.

5. Loube DI, Gay PC, Strohl KP, Pack AI, White DP, Collop NA. Indications for positive airway pressure treatment of adult obstructive sleep apnea patients: a consensus statement. Chest 1999;115: 863-6.

6. Appels A, Hoppener P, Mulder P. A questionnaire to assess premonitory symptoms of myocardial infarction. Int J Cardiol 1987; 17: 1524.

7. Johns MW. Re thinking the assessment of sleepiness. Sleep Med Rev 1998; 2: 3-15.

8. Kales, Caldwell, A.Cadieux, R, et al Severe obstructive sleep apnea - II: Associated psychological and psychosocial consequences. J Chronic Dis 1985; 38: 426437.

9. Greenberg G, Watson R, Deptula D. Neuropsychological dysfunction in sleep apnea.

Sleep 1987; 10: 254-262.

10. Tousignant P, Cosio M, Levy R, et al. Quality adjusted life years added by treatment of obstructive sleep apnea. Sleep 1994; 17: 52-60.

11. D'Ambrosio C, Bowman T, and Mohsenin V. Quality of Life in Patients with Obstructive Sleep Apnea. Effect of Nasal Continuous Positive Airway Pressure-A Prospective Study. Chest 1999; 115: 123-129. 